\title{
Definition and test of the electromagnetic immunity of UAS for first responders
}

\author{
C. Adami, S. Chmel, M. Jöster, T. Pusch, and M. Suhrke \\ Fraunhofer Institute for Technological Trend Analysis (INT), Euskirchen, Germany \\ Correspondence to: M. Jöster (michael.joester@int.fraunhofer.de)
}

Received: 10 December 2014 - Revised: 24 April 2015 - Accepted: 22 May 2015 - Published: 3 November 2015

\begin{abstract}
Recent technological developments considerably lowered the barrier for unmanned aerial systems (UAS) to be employed in a variety of usage scenarios, comprising live video transmission from otherwise inaccessible vantage points. As an example, in the French-German ANCHORS project several UAS guided by swarm intelligence provide aerial views and environmental data of a disaster site while deploying an ad-hoc communication network for first responders. Since being able to operate in harsh environmental conditions is a key feature, the immunity of the UAS against radio frequency (RF) exposure has been studied. Conventional Electromagnetic Compatibility (EMC) applied to commercial and industrial electronics is not sufficient since UAS are airborne and can as such move beyond the bounds within which RF exposure is usually limited by regulatory measures. Therefore, the EMC requirements have been complemented by a set of specific RF test frequencies and parameters where strong sources are expected to interfere in the example project test case of an inland port environment. While no essential malfunctions could be observed up to field strengths of $30 \mathrm{~V} \mathrm{~m}^{-1}$, a sophisticated, more exhaustive approach for testing against potential sources of interference in key scenarios of UAS usage should be derived from our present findings.
\end{abstract}

\section{Introduction}

Advances in technology allow for sophisticated, unmanned robots and vehicles to explore environments otherwise inaccessible to human investigation due to hazardous conditions like high temperatures, noxious chemicals or ionizing radiation exceeding safety thresholds. In case of large scale disasters, obstructions on ground level may point to aerial reconnaissance as a viable option. The use of single UAS equipped with sensor systems has already seen some application (Pölläna et al., 2009). In order to quickly and continuously chart a disaster site, operating several robotic systems in parallel is necessary. Coordination schemes for multiple UAS in joint operation have been investigated (Simi et al., 2013). There are attempts at controlling clouds of air contaminants by UAS networks (White et al., 2008). Projects like SENEKA involve unmanned ground systems (UGS) in conjunction with UAS (Kuntze et al., 2012). The same is true for the ANCHORS (UAV-Assisted Ad Hoc Networks for Crisis Management and Hostile Environment Sensing) project where a special focus was laid on detection of ionizing radiation (Berky et al., 2014). Within this project running 2012-2015, a system is developed to combine UAS into an autonomous swarm of sensors while at the same time providing a dynamic communication infrastructure of digital radio network cells.

When relying on such systems in order to improve situational awareness of first responders, robustness against any disturbance is crucial. Depending on the deployment scenario, the UAS will have to withstand electromagnetic interference (EMI) by strong RF sources. In previous studies, UAS operation in close proximity to radio broadcasting station has been investigated (Torrero et al., 2013). Other studies have been focussing on actually avoiding strong radar sources by flight path planning algorithms (Chen et al., 2014; Duan et al., 2014).

Radar sources may be prevalent when considering logistics, such as cargo processing in airports and ports. As an example, the ANCHORS project test case conceives a strong industrial source of ionising radiation averaging in the port of Dortmund, Germany. In this case, port radar and ship radar transmitters would have to be taken into account. 


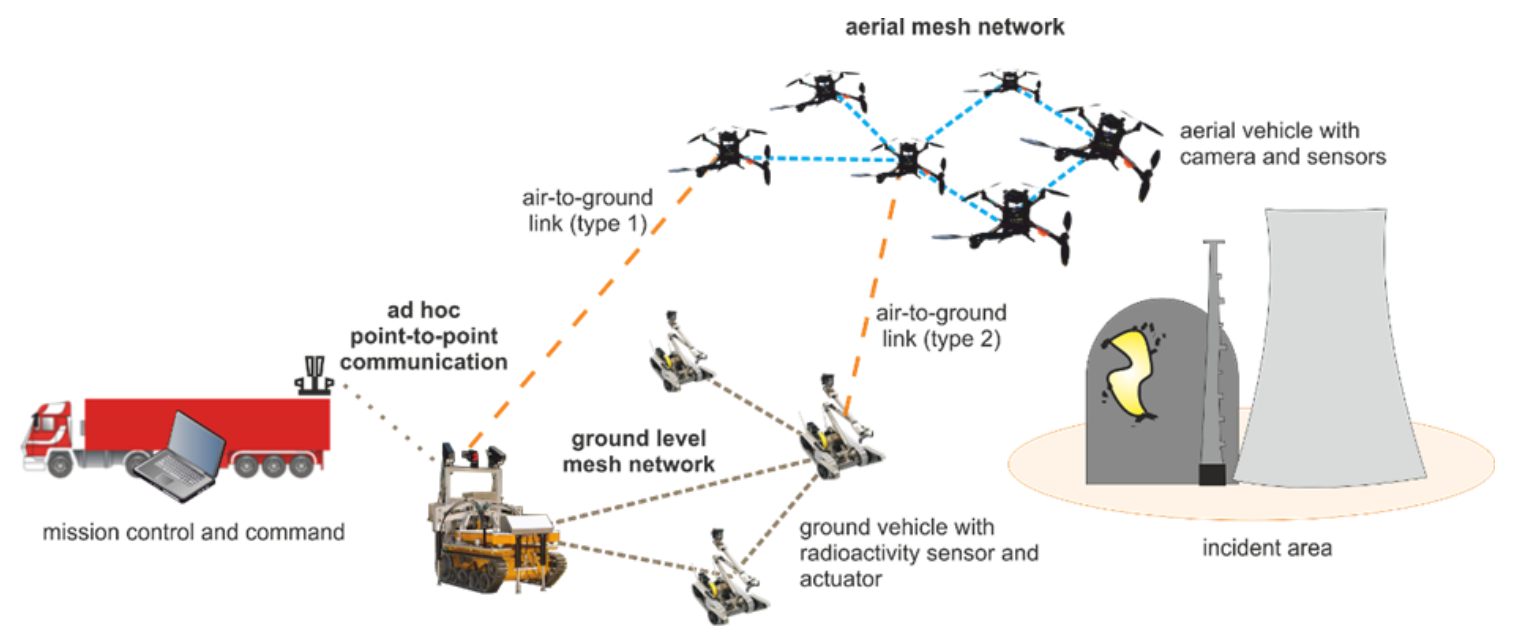

Figure 1. ANCHORS: All actors of the autonomous systems including network connections during an incident (Source: ANCHORS Consortium).

In addition to flight stability and operability, the wireless data link to base stations represents an essential part of the system functionality. Potential disruptions have been subject to research as well (Zhang et al., 2013; Guo et al., 2013).

In our present study, we address the issue of electromagnetic compatibility (EMC) of one of the ANCHORS UAS. We expand on a classic EMC test procedure (DIN Deutsches Institut für Normung e.V., 2006) by selecting additional RF frequencies and test parameters based on the test case scenario analysis. We will present details of the ANCHORS system concept in order to assess potential vulnerabilities, as well as details about the employed test methods and the diagnostics setup. In our tests ranging up to field strengths of $30 \mathrm{~V} \mathrm{~m}^{-1}$, no essential malfunctions could be observed. Nevertheless, we deem further research to be essential. We do point out some starting points in our conclusion, expanding on the scenario-based approach.

\section{Description of the ANCHORS system concept}

The ANCHORS concept foresees several UAS deployed by a UGS base station in the immediate vicinity of a disaster site. In Fig. 1, the UAS coalescing to a self-regulated swarm are depicted. They communicate with each other via radio, allowing for a flexible role management system where the swarm dynamically accommodates for vacated network nodes. These occur during power replenishment or decontamination actions the UAS perform automatically by autonomous landing on a mobile UGS base station.

All swarm members can be variably equipped with cameras or radiation sensors and send the collected data via radio network to the incident command.

In addition, the UAS are airborne relay stations for radio network cells in ad-hoc communications. As shown in Fig. 2, groups of response personnel are provided with a local net- work cell which combines with other relay stations, local incident command and possibly remote headquarters to an allencompassing network. The wireless standards "Long Term Evolution" (LTE) and "Private Mobile Radio" (PMR) have been settled upon for communications.

\section{Analysis of the electromagnetic environment of the swarm system}

\subsection{Common aspects of typical usage scenarios}

The whole system of mobile and stationary swarm members as shown in Fig. 2 might be influenced by the local electromagnetic environment of the operational area. In a first step, some assumptions common to typical environments can be made. Because of the high coverage of mobile services in many countries, stationary base stations are part of this electromagnetic environment with a high probability.

Wireless communication and IT devices with relatively low transmitter power are common in residential zones. In industrial zones, we expect a considerable variety of businessspecific transmitters like local wireless communication and IT networks with low transmitter power, broadcast stations with medium and high power, and stationary as well as mobile radar facilities in harbors, on ships, at airports and on aircrafts with high and very high pulsed power. Even electromagnetic interferences by unintentional RF transmissions are possible in industrial areas, as generated for example by power inverters.

Moreover, usual protection measures for persons against high field values generated by stationary transmitters by keeping them at distance by structural measures like fences do not work for UAS automatically. 


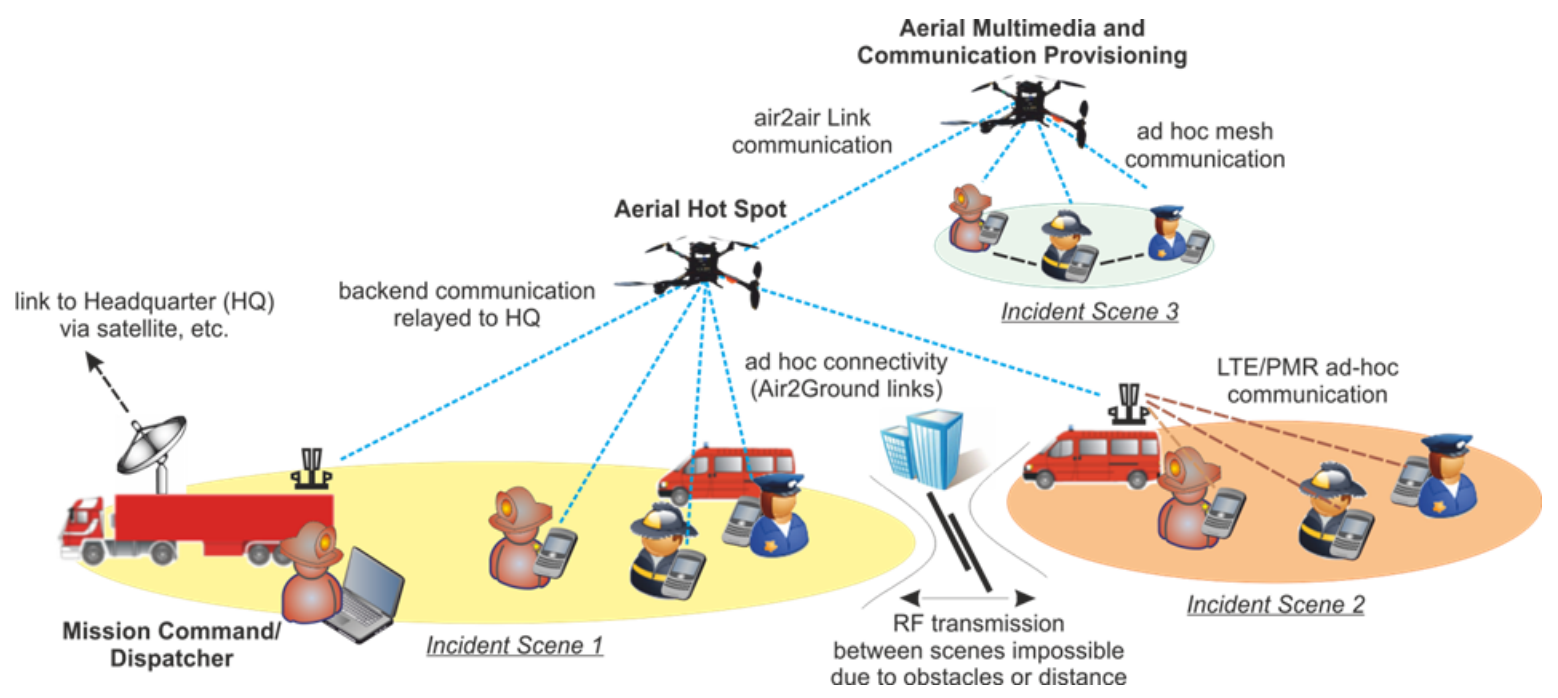

Figure 2. ANCHORS: UAVs as relay stations in an ad-hoc communications radio network (Source: ANCHORS consortium).

\subsection{The ANCHORS test case environment}

In the following, we will refer to the test case scenario of the ANCHORS project as an example for a potential application case for UAS. We have compiled a comprehensive list of RF sources possibly interfering with system functionality. This includes a suggestion for a geographical analysis of RF exposure based upon which further measures could be developed, like no-fly areas to be integrated into the system software.

As a part of the ANCHORS project, a large scale incident scenario in the harbor of Dortmund has been developed. Two radioactive sources used for on-site material inspection have sprung a leak during an accident and radioactive material is released in the environment.

With regard to the specified location, a mobile network base station has been identified in the direct incident area of the scenario. Figure 3 a shows this location on a map of the harbor.

In Germany, information about such base stations is available in a public database (Federal Network Agency, http: //emf3.bundesnetzagentur.de/karte/Default.aspx) and can be used for location-based RF exposure assessment. A safety distance for each sector antenna is given, at which the electric field strength meets the limits for human exposure given in the related German electromagnetic fields regulations (BMUB, 2013). As the transmitter frequencies are not accessible in the database, whereas the field strength limit is frequency dependent, the electric field limit value at the indicated safety distance has been calculated back to $1 \mathrm{~m}$ in front of the antenna for all possible network service frequencies. As a worst case estimate, the highest value of field strength per antenna has been taken to calculate the distance needed to keep electric fields below $E=10 \mathrm{~V} \mathrm{~m}^{-1}$. The area within this safety perimeter has been shaded in red in Fig. $3 \mathrm{~b}$.
The field strength of $E_{\mathrm{CE}}=10 \mathrm{~V} \mathrm{~m}^{-1}$ represents the EMC immunity required by EU regulations for electronic equipment used in industrial environments (BMUB, 2013), valid for LTE800 and GSM900 mobile networks. For services using frequencies in the range 1.4 up to $2 \mathrm{GHz}$, like GSM1800 and LTE1900, the tested severity level is just $E_{\mathrm{CE}}=3 \mathrm{~V} \mathrm{~m}^{-1}$. Above $2 \mathrm{GHz}$ where services like UMTS and LTE2600 are located the tested level is lowered to $E_{\mathrm{CE}}=1 \mathrm{~V} \mathrm{~m}^{-1}$. The EMC immunity performance of typical commercial UAS beyond these test levels is most probably unknown for lack of any additional conformity requirements taking the extended spatial mobility into account.

When maintaining a rather cautious stance regarding system safety, flight paths infringing the red-marked area in Fig. 4b have to be avoided in case the network service GSM900 is installed at the mobile base station. The required distance is up to $55 \mathrm{~m}$. In most cases, even more network services are provided by a single base station, so the safety distance will be much higher with 183 and $549 \mathrm{~m}$ relating to the two lower test levels of 3 and $1 \mathrm{~V} \mathrm{~m}^{-1}$ in the related frequency bands, respectively.

When considering the electromagnetic environment of the ANCHORS scenario, it can be assumed that there are radar facilities present, mobile ones on ships and a stationary one in the port area. Additionally, a scenario-independent source of electromagnetic energy is inherent in the ANCHORS concept, the ad-hoc network capability. To realize an airborne network relay, a transmitter will be mounted directly underneath each UAS. To complete the list of electromagnetic sources in the environment, there are transmitters for wireless communication and UAS/UGS remote control located in the safe boundary area where the first responders act on ground. 


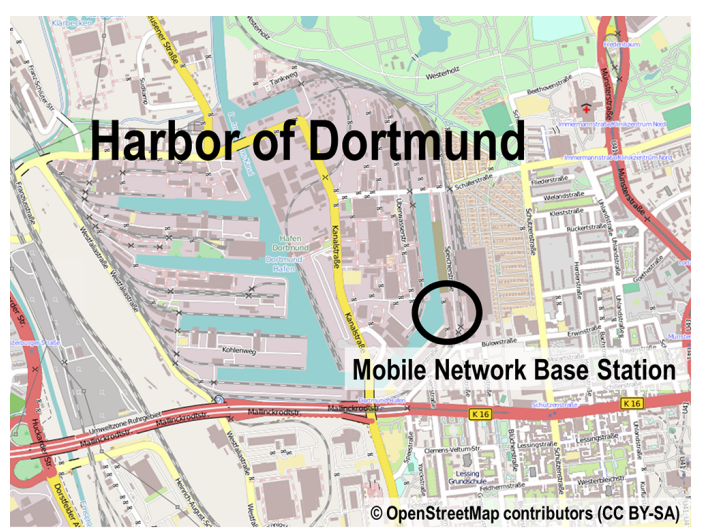

(a)

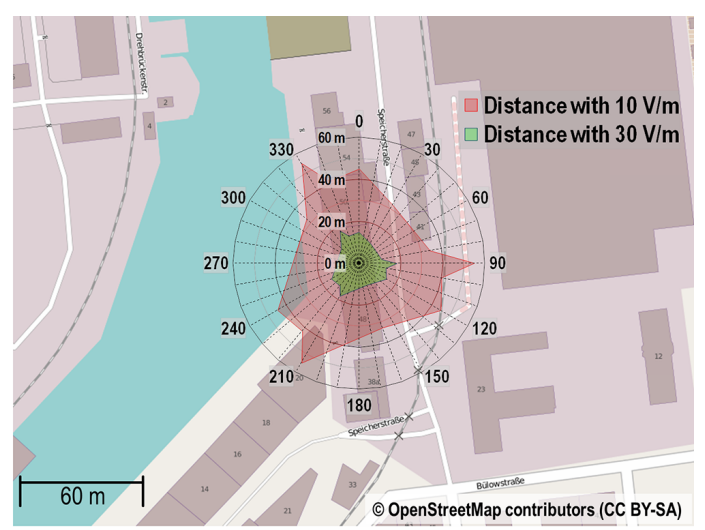

(b)

Figure 3. (a) Location of a mobile network service base station within the direct incident area of the scenario developed in the ANCHORS project. (b) Calculated areas of $E \geq 10 \mathrm{~V} \mathrm{~m}^{-1}$ (red color) and $E \geq 30 \mathrm{~V} \mathrm{~m}^{-1}$ (green color) around the mobile network base station (source of maps: http://openstreetmap.org).

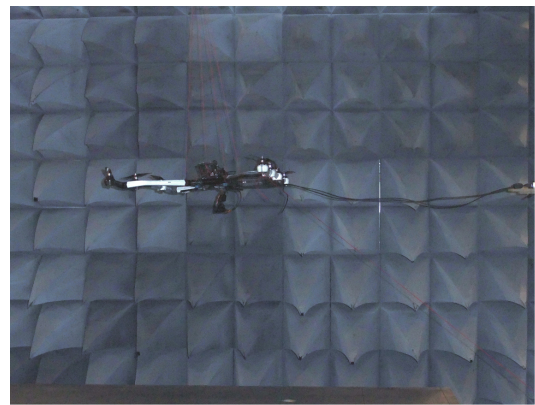

(a)

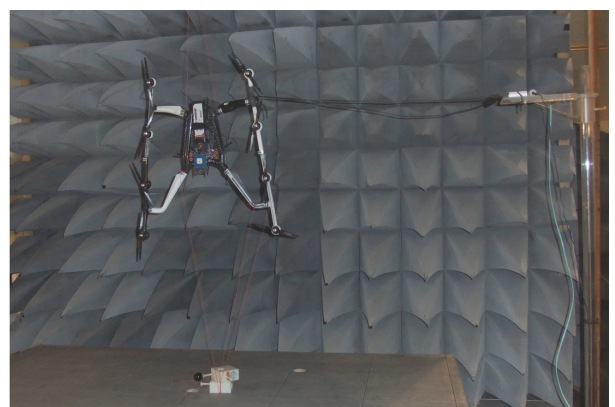

(b)

Figure 4. (a) Horizontal and (b) vertical test positions of the UAS in the TEM waveguide.

\section{Elaboration of parameters for laboratory testing}

To address a common susceptibility risk of the entire ANCHORS system, a basic normative immunity test has been defined according DIN EN 61000-6-2 (DIN Deutsches Institut für Normung e.V., 2006), covering the range of most technically used frequencies. This test standard covers the frequency range from 80 to $2700 \mathrm{MHz}$ with electrical field strength test values from $E=10 \mathrm{~V} \mathrm{~m}^{-1}$ down to $1 \mathrm{~V} \mathrm{~m}^{-1}$, as detailed in the previous section. When testing with these parameters, no relevant function of the Device Under Test (DUT) is allowed to degrade. As the key result of the scenario-related electromagnetic environment analysis this basic immunity requirement has been expanded by a set of frequencies where the DUT is expected to be particularly vulnerable. Table 1 gives an overview of all tested frequencies.

All additional frequencies have been tested with $E=30 \mathrm{~V} \mathrm{~m}^{-1}$, the highest defined test level of the test standard (DIN Deutsches Institut für Normung e.V., 2006). Figure $4 \mathrm{~b}$ shows a green area where the field strength exceeds $30 \mathrm{~V} \mathrm{~m}^{-1}$ for the mobile network services provided by this base station. The maximum distance a UAS immune to this field strength level shall observe with regard to the base station reduces from $55 \mathrm{~m}$ in case of the previously mentioned $10 \mathrm{~V} \mathrm{~m}^{-1}$ limit to $18 \mathrm{~m}$.

To simulate the digital modulation, the GSM time slot pulse with $570 \mu$ s length and $4.6 \mathrm{~ms}$ pulse repetition time has been taken as basis for all communication frequencies. Radar signals are simulated by pulses with $1 \mu$ s length and $1 \mathrm{~ms}$ pulse repetition time. An open TEM waveguide has been used for normative immunity testing according DIN EN 61000-4-20.

\section{Test setup and diagnostics}

The EMC immunity test standard our study refers to (DIN Deutsches Institut für Normung e.V., 2006), classifies temporary and permanent degradation of functionality in steps of usability of the DUT. Four classes are defined, ranging from "a" for no degradation during RF exposure to "d" for permanent malfunction after exposure. The functional degradation a UAS can cope with is obviously limited, as many functions have a direct influence on navigation and flight sta- 
Table 1. Specified test frequencies for the ANCHORS project as derived from scenario.

\begin{tabular}{llc}
\hline Frequency & Service & Immunity test value \\
\hline $80-1000 \mathrm{MHz}$ & Basic EMC immunity requirement & $10 \mathrm{~V} \mathrm{~m}^{-1}$ \\
$1400-2000 \mathrm{MHz}$ & Basic EMC immunity requirement & $3 \mathrm{~V} \mathrm{~m}^{-1}$ \\
$2000-2700 \mathrm{MHz}$ & Basic EMC immunity requirement & $1 \mathrm{~V} \mathrm{~m}^{-1}$ \\
$400 \mathrm{MHz}$ & LTE/PMR communication within ANCHORS, on-board transmitter & $30 \mathrm{~V} \mathrm{~m}^{-1}$ \\
$2400 \mathrm{MHz}$ & Remote control, other services on 2.4 GHz ISM (Industrial, Scientific, and Medical) band & $30 \mathrm{~V} \mathrm{~m}^{-1}$ \\
$5200 \mathrm{MHz}$, & UAS remote control downlink channel, & $30 \mathrm{~V} \mathrm{~m}^{-1}$ \\
$5800 \mathrm{MHz}$ & other services on 5 GHz ISM band & $30 \mathrm{~V} \mathrm{~m}^{-1}$ \\
$810 \mathrm{MHz}$, & GSM/LTE stationary base stations, & \\
$2660 \mathrm{MHz}$, & ANCHORS LTE/PMR communication & $30 \mathrm{~V} \mathrm{~m}^{-1}$ \\
$1840 \mathrm{MHz}$ & with on-board transmitter & \\
$3020 \mathrm{MHz}$, & Stationary and mobile naval radar facilities & \\
$9375 \mathrm{MHz}$ & in S- and X-band & \\
\hline
\end{tabular}

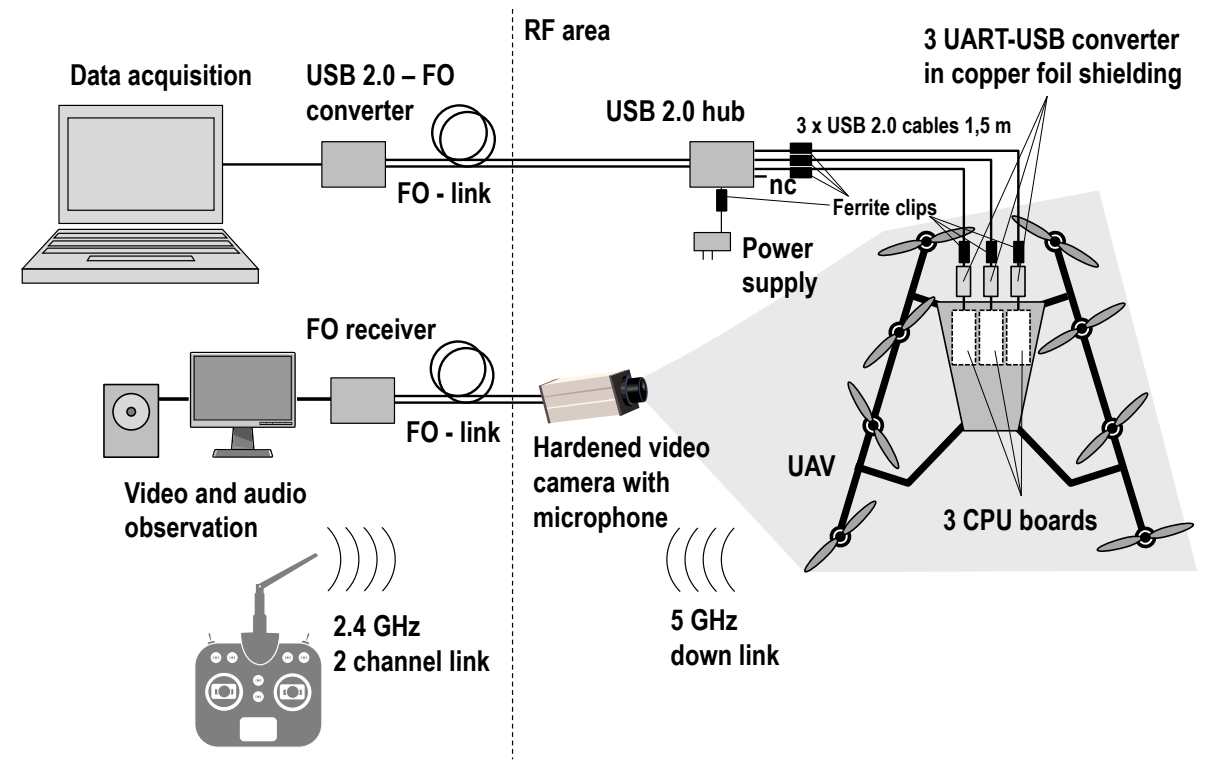

Figure 5. Overview sketch of the monitoring test setup.

bilization. Therefore the flight relevant functions have been identified and assigned to the normative performance class "a". In case of the ANCHORS UAS, some basic functions are designed redundantly in the system. While the respective functional unit as a whole is required to comply with performance class "a" criteria, substructures may degrade to performance class "b", designating a temporary malfunction during RF exposure. As an example, a certain number of the four motor drivers per side may fail and the UAS will nevertheless remain in a stable flight condition.

The functionality classification being complete, the functions identified are described with electrical parameters and tolerances, which have to be monitored during testing. The UAS has been tested in horizontal and vertical position within the open TEM waveguide as it is shown in Fig. 4. The data stream allowing the monitoring of the relevant functions passed a fiber optic link to a monitoring PC outside of the RF test hall.

Figure 5 gives an overview of the monitoring test setup. By a hardened video camera with microphone the revolution speed of the rotors could be observed.

The tests have been performed with the substitution method described in the test standard. After calibrating the empty TEM waveguide to the desired electric field value at each test frequency, the stored forward power values are then provided again during a test run with the DUT in place. The power adjustment tolerance foresees an appropriate slight over-testing. Figure 6 gives an overview of the RF test site used for the immunity tests. 


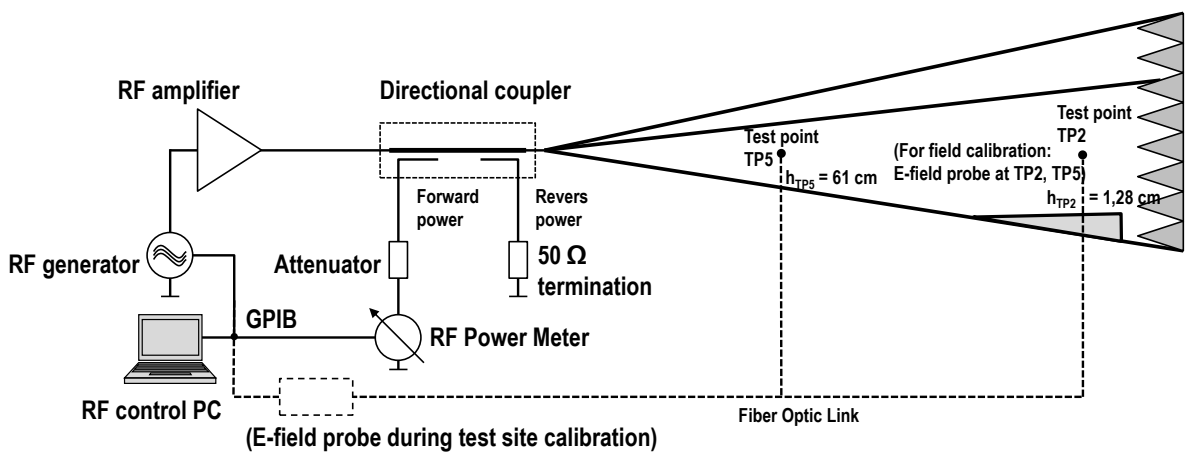

Figure 6. Overview sketch of the RF test setup based on the open TEM waveguide.

Table 2. Test result summary.

\begin{tabular}{|c|c|c|c|c|}
\hline Frequency & Test parameter* & Observed effects & $\begin{array}{l}\text { Performance class } \\
\text { required/reached }\end{array}$ & Evaluation \\
\hline $80 \mathrm{MHz}-1 \mathrm{GHz}$ & $10 \mathrm{~V} \mathrm{~m}^{-1}, \mathrm{AM}$ & none & $\mathrm{a} / \mathrm{a}$ & pass \\
\hline $1.4-2 \mathrm{GHz}$ & $3 \mathrm{Vm}^{-1}, \mathrm{AM}$ & $\begin{array}{l}2.4 \mathrm{GHz} \text { remote control link disturbed } \\
\text { between } 1887-2002 \mathrm{MHz}\end{array}$ & $\mathrm{b} / \mathrm{b}$ & pass \\
\hline $2-2.7 \mathrm{GHz}$ & $10 \mathrm{~V} \mathrm{~m}^{-1}, \mathrm{AM}$ & $\begin{array}{l}2.4 \mathrm{GHz} \text { remote control link disturbed } \\
\text { between } 2040-2616 \mathrm{MHz}\end{array}$ & $\mathrm{b} / \mathrm{b}$ & pass \\
\hline $400 \mathrm{MHz}$ & $30 \mathrm{~V} \mathrm{~m}^{-1}, \mathrm{GSM}$ & none & $\mathrm{a} / \mathrm{a}$ & pass \\
\hline $2400 \mathrm{MHz}$ & $30 \mathrm{~V} \mathrm{~m}^{-1}, \mathrm{GSM}$ & none & $\mathrm{a} / \mathrm{a}$ & pass \\
\hline $\begin{array}{l}5200 \mathrm{MHz}, \\
5800 \mathrm{Mhz}\end{array}$ & $30 \mathrm{~V} \mathrm{~m}^{-1}, \mathrm{GSM}$ & none & $\mathrm{a} / \mathrm{a}$ & pass \\
\hline $\begin{array}{l}810 \mathrm{MHz}, \\
1840 \mathrm{MHz}, \\
2660 \mathrm{MHz}\end{array}$ & $30 \mathrm{~V} \mathrm{~m}^{-1}, \mathrm{GSM}$ & none & $\mathrm{a} / \mathrm{a}$ & pass \\
\hline $\begin{array}{l}3020 \mathrm{MHz} \\
9375 \mathrm{MHz}\end{array}$ & $30 \mathrm{~V} \mathrm{~m}^{-1}$, Pulse & none & $\mathrm{a} / \mathrm{a}$ & pass \\
\hline
\end{tabular}

* $\mathrm{AM}=$ Amplitude modulation $80 \%, 1 \mathrm{kHz} ; \mathrm{GSM}=$ Pulse modulation $570 \mu \mathrm{s} / 4.6 \mathrm{~ms} ;$ Pulse $=$ Pulse modulation $1 \mu \mathrm{s} / 1 \mathrm{~ms}$.

\section{Test results}

As Table 2 reflects, the UAS fulfilled all requirements fixed in Sect. 4.

As predicted and considered in the test requirements, one of two redundant remote control links in the $2.4 \mathrm{GHz}$ band quit during exposure to test pulses at frequencies within this ISM band. Surprisingly the same issue occurred with test frequencies between 1880 and $2400 \mathrm{MHz}$, and 2500 and $2620 \mathrm{MHz}$, this might be related to an issue with band filtering in the $2.4 \mathrm{GHz}$ receiver module. As the remote link is still operational with the remaining link channel, the required functional state is fulfilled. By an oversight related to the RF test setup, the frequency range of 1400 up to $2700 \mathrm{MHz}$ has been slightly over-tested with a minimum factor of two, but the DUT kept full functionality. Some additional tests have been performed in order to probe the DUT at even higher field strengths. The electronic design of the UAS shows a good immunity margin up to $80 \mathrm{~V} \mathrm{~m}^{-1}$, as the UAS still fulfilled the functional performance requirements.

\section{Conclusion}

Battery technologies with high energy density and high computing power in small and light weight units made UAS to very appealing devices to be used for aerial reconnaissance in crisis situations, where the direct incident area is not accessible by humans anymore. But during a mission, they can enter areas of high electromagnetic fields, typically controlled against access by structural measures taking no aerial approach at low altitudes into account. These electromagnetic fields are larger than the EMC immunity standard levels consumer and industrial electronics are designed for, derived from the electromagnetic environment in normal daily usage situations. Therefore, the issue of RF immunity to high level exposure has to be addressed by users of UAS in critical missions where the reliability of the devices has to be high.

In this work, a baseline electromagnetic environment has been estimated for residential and industrial areas. With a very high probability, a mobile network base station will be part of it. In order to focus on usage of UAS in large scale 
incidents, a scenario at a real location in Germany has been defined within the ANCHORS project. Analysis of the scenario led to a definition of basic and additional test frequencies. As a project task, the RF immunity of the UAS has been evaluated applying the EMC immunity test standard for industrial electronic devices. The UAS is immune to the set of selected frequencies and showed an immunity margin up to a minimum factor of two compared to the normative test levels.

Further hardening measures to increase the immunity especially at mobile network service frequencies might be undertaken, but will possibly reduce the amount of payload, flight duration and mission range of the UAS by increasing weight. As a hybrid solution, immunization measures could be combined with a map indicating keep-out areas around stationary transmitters like mobile network base stations, broadcast stations and radar stations. Thus, the UAS could navigate around areas with high RF field strengths.

In ANCHORS, a key capacity in development is the swarm capability. As the devices interact with each other, the whole swarm can be seen as a controlled system. The behavior of each member in association with others in the swarm can be conceived as control loops, reacting on expected disturbances like wind, but to as of yet unexpected electromagnetic disturbances, too. Suppressed communication can break the swarm as well as corrupted or missing navigation and position information of single swarm members.

Acknowledgements. The project ANCHORS is sponsored by the German Federal Ministry of Education and Research (BMBF) and the French National Research Agency (ANR).

The authors thank the company Ascending Technologies for the close cooperation during setup preparation and tests.

Edited by: F. Gronwald

Reviewed by: H. Garbe and one anonymous referee

\section{References}

Berky, W., Chmel, S., Friedrich, H., Höffgen, S. K., Jöster, M., Köble, T., Lennartz, W., Metzger, S., Pusch, T., Risse, M., Schumann, O., Rosenstock, W., and Weinand, U.: Air-Bound Measurements of Radioactive Material with Swarm-Behaved UAVs The ANCHORS Project, Proc. 9th Future Security Conference, Berlin, Germany, 16-18 September 2014, 64-70, 2014.
DIN Deutsches Institut für Normung e.V.: DIN EN 61000-6-2: Electromagnetic compatibility (EMC) - Part 6-2: Generic standards - Immunity for industrial environments (IEC 61000-62:2005), 2006.

Duan, Y., Ji, X., Li, M., and Li, Y.: Route planning method design for UAV under radar ECM scenario, 12th International Conference on Signal Processing (ICSP), Hangzhou, China, 19-23 October 2014, 108-114, doi:10.1109/ICOSP.2014.7014979, 2014.

Federal Ministry for the Environment, Nature Conservation, Building and Nuclear Safety (BMUB): 26th BImSchV, Amended ordinance on electromagnetic fields (14 August 2013), 3266, 2013.

Gao, C., Gong, H., Zhen, Z., Zhao, Q., and Sun, Y.: Three dimensions formation flight path planning under radar threatening environment, 33rd Chinese Control Conference (CCC), Nanjing, China, 28-30 July 2014, 1121-1125, doi:10.1109/ChiCC.2014.6896785, 2014.

Guo, S., Dong, Z., Hu, Z., and Hu, C.: Simulation of dynamic electromagnetic interference environment for Unmanned Aerial Vehicle data link, China Communications, 10, 19-28, doi:10.1109/CC.2013.6570796, 2013.

Kuntze, H., Frey, C. W., Tchouchenkov, I., Staehle, B., Rome, E., Pfeiffer, K., Wenzel, A., and Wollenstein, J.: SENEKA - sensor network with mobile robots for disaster management, IEEE Conference on Technologies for Homeland Security (HST), Waltham, MA, USA, 13-15 November 2012, 406-410, doi:10.1109/THS.2012.6459883, 2012.

Pölläna, R., Peräjärvia, K., Karhunena, T., Ilandera, T., Lehtinenb, J., Rintalac, K., Katajainenc, T., Niemeläc, J., and Juuselac, M.: Radiation surveillance using an unmanned aerial vehicle, Appl. Rad. Iso., 67, 340-344, doi:10.1016/j.apradiso.2008.10.008, 2009.

Simi, S., Kurup, R., and Rao, S.: Distributed task allocation and coordination scheme for a multi-UAV sensor network, Tenth International Conference on Wireless and Optical Communications Networks (WOCN), Bhopal, India, 26-28 July 2013, 1-5, doi:10.1109/WOCN.2013.6616189, 2013.

Torrero, L., Mollo, P., Molino, A., and Perotti, A.: RF immunity testing of an Unmanned Aerial Vehicle platform under strong EM field conditions, 7th European Conference on Antennas and Propagation (EuCAP), Gothenburg, Sweden, 8-12 April 2013, 263-267, 2013.

White, B. A., Tsourdos, A., Ashokaraj, I., Subchan, S., and Zbikowski, R.: Contaminant Cloud Boundary Monitoring Using Network of UAV Sensors, IEEE Sensors Journal, 8, 1681-1692, doi:10.1109/JSEN.2008.2004298, 2008.

Zhang, T., Chen Y., and Cheng E.: Continuous wave radiation effects on UAV data link system, Cross Strait QuadRegional Radio Science and Wireless Technology Conference (CSQRWC), Chengdu, China, 21-25 July 2013, 321-324, doi:10.1109/CSQRWC.2013.6657419, 2013. 